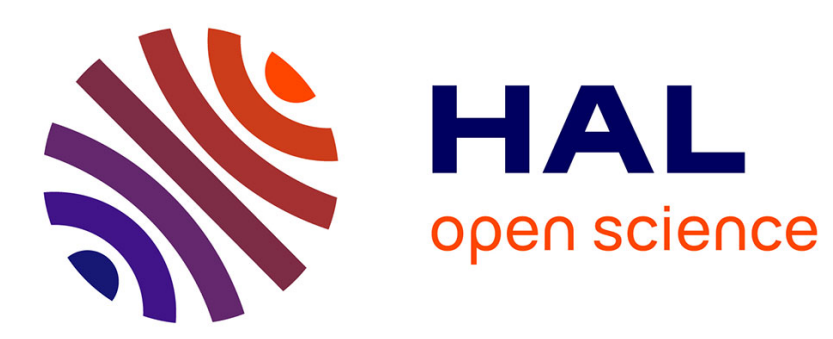

\title{
A Vision/Position/Force Control Approach for Performing Assembly Tasks with a Humanoid Robot
}

Pierre Dauchez, Philippe Fraisse, François Pierrot

\section{To cite this version:}

Pierre Dauchez, Philippe Fraisse, François Pierrot. A Vision/Position/Force Control Approach for Performing Assembly Tasks with a Humanoid Robot. Humanoids, Dec 2005, Tsukuba, Japan. lirmm00106499

\section{HAL Id: lirmm-00106499 \\ https://hal-lirmm.ccsd.cnrs.fr/lirmm-00106499}

Submitted on 16 Oct 2006

HAL is a multi-disciplinary open access archive for the deposit and dissemination of scientific research documents, whether they are published or not. The documents may come from teaching and research institutions in France or abroad, or from public or private research centers.
L'archive ouverte pluridisciplinaire HAL, est destinée au dépôt et à la diffusion de documents scientifiques de niveau recherche, publiés ou non, émanant des établissements d'enseignement et de recherche français ou étrangers, des laboratoires publics ou privés. 


\section{A Vision/Position/Force Control Approach for Performing Assembly Tasks with a Humanoid Robot}

\author{
Pierre Dauchez \\ LESIA/INSA Toulouse - EA 1687 du MENESR \\ 135 Avenue de Rangueil \\ 31077 Toulouse CEDEX 4, France \\ pierre.dauchez@insa-toulouse.fr
}

\author{
Philippe Fraisse, François Pierrot \\ LIRMM - UMR 5506 CNRS/Université Montpellier II \\ 161 rue Ada \\ 34392 Montpellier CEDEX 5, France \\ philippe.fraisse@lirmm.fr, francois.pierrot@lirmm.fr
}

\begin{abstract}
Humanoid robots are currently widely studied because of the many future applications they could be used for. Many researchers are working on the locomotion aspects. We are more interested in dual-arm manipulation tasks, because of our long experience on this topic. In this paper, we consider the task consisting in assembling two objects, each of them being held by one of the humanoid arms, as human beings often do. In this framework, we briefly recall methods that we have developed in the past for (i) hybrid position/force control of a two-arm robot and (ii) relative positioning of the end-effectors of two manipulators. We propose to combine these approaches with a solution using the vision system of the humanoid in order to fully automatize the assembly task. Tests have successfully been performed with the OpenHRP simulator of the HRP2 humanoid but they only represent preliminary work. Therefore, this short paper should be considered as an introduction to the research activities we would like to focus on in the future.
\end{abstract}

Index Terms - Humanoid, Manipulation Tasks, Vision/Position/Force Control.

\section{INTRODUCTION}

For several years now, many research laboratories all over the world have been interested in humanoid robots. The focus of their work has mainly been on developing very impressive prototypes integrating many robotics features, on planning collision-free movements or on studying the stability of bipeds using various gaits.

On the opposite, it seems that less researchers were interested in the control of the humanoid's arms, perhaps because it was thought that the solutions developed for nonhumanoid multi-arm systems could be used as are. Since the authors of this paper have a very long experience in control of two-arm robots (since 1981), it came obvious to them to extend their work to the humanoid case. This is the framework of this paper where the generic task that is considered is the automatic assembly of two objects, each of them being held by one arm, using the stereoscopic vision system of the humanoid and position/force control of the arms.

The robot that is considered for simulation purpose is the Japanese HRP2 humanoid (Fig. 1) [1], because of the close connections and cooperative work between the LIRMM and the JRL (Joint Japanese-French Robotics Laboratory) since the creation of the latter.
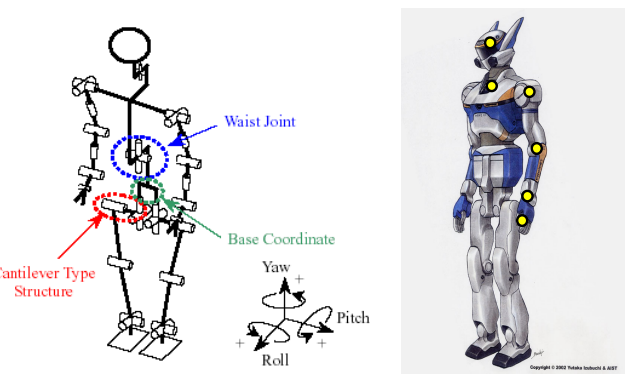

Fig. 1 Sketches of the HRP2 humanoid robot

Section II briefly summarizes a position/force control method that has initially been developed to manipulate rigid objects with two arms and explains how it can be used for an assembly task. The authors are perfectly aware that many other developments related to multi-manipulator systems have occurred in the world since the 80's. As a matter of fact, they have discussed with most of the researchers involved in the field and have organized many special sessions on this topic in international conferences. This is why it is important to understand that their project is built upon solutions they have implemented and successfully tested with real robots, but does not reject any other approach, that is not referenced here.

Section III shows how we can automatically bring the two objects in contact (approach phase of an assembly task) by considering the two arms as a single manipulator.

These two methods have been proposed in the 80's and then widely tested in simulation and with real robots. Therefore, rather than describing them again in details, we focus here on the general ideas and on the motivation behind these developments. The reason why they are recalled here is that we strongly believe that they could be implemented on a real humanoid robot for performing manipulation tasks similar to what human beings do.

But this would require the help of a vision system and this is why Section IV introduces the use of the humanoid "eyes" to detect the positions of the objects held by the arms. The combination of these methods should allow a real system to automatically perform the assembly of two objects. For the 
moment, only simulations related to the relative motion of the arms and to the head/arms positioning have been performed for a HRP2 humanoid. The results are not reported here because of their non-relevancy to the purpose of the paper.

\section{ASSEMBLY OF TWO OBJECTS USING TWO POSITION/FORCE CONTROLLED MANIPULATORS}

\section{A. General Approach}

Pioneer work on hybrid position/force control of robot manipulators is probably that of Raibert and Craig [2]. This principle was then extended to two-arm systems [3, 4] (for instance). We briefly recall here the idea proposed by Uchiyama and Dauchez in [3] as it can be used for the assembly task considered in this paper.

Their method is based upon the initial idea to consider a two-arm robot as a unique system and to develop a symmetric hybrid position/force control solution that considers the object as the central focus point of the system in the case of two robots carrying a rigid object, as a human being probably does. Then the variables to control would be the absolute position/orientation of the object in space, the relative position/orientation of the end-effectors, the external force/moment exerted on the object and the internal force/moment in the object. To mathematically determine these entities, which is necessary to introduce them in a control loop, so-called "virtual sticks" are introduced. They are rigid segments that initially link the centers $\mathbf{O}_{\mathbf{h} 1}$ and $\mathbf{O}_{\mathbf{h} 2}$ of the end-effectors to a user-chosen reference point $\mathbf{O}_{\mathbf{a}}$ attached to the object (not necessarily on the object) and allow to drastically simplify the force/torque relationships, assuming a small discrepancy between the tips of the sticks during any motion. Hence the velocity and position/orientation equations between what happens at the arms level and what happens at the object level can be derived and two control vectors can be defined, both related to the object [3]:

$$
\mathbf{z}=\left(\begin{array}{l}
\mathbf{p a} \\
\mathbf{p r}
\end{array}\right) \text { and } \mathbf{h}=\left(\begin{array}{l}
\mathbf{f}_{\mathbf{a}} \\
\mathbf{f} \mathbf{r}
\end{array}\right)
$$

$\mathbf{p a}_{\mathbf{a}}$ represents the absolute position/orientation of the object (average position/orientation of the tips of the virtual sticks), $\mathbf{p}_{\mathbf{r}}$ represents the relative position/orientation of the endeffectors (difference between the positions/orientations of the tips of the virtual sticks), $\mathbf{f}_{\mathbf{a}}$ represents the external force/moment acting on the object (corresponding to $\mathbf{p}_{\mathbf{a}}$ ), $\mathbf{f}_{\mathbf{r}}$ represents the internal force/moment in the object (corresponding to $\mathbf{p}_{\mathbf{r}}$ ). $\mathbf{p}_{\mathbf{a}}$ and $\mathbf{f}_{\mathbf{a}}$ have the same dimension. $\mathbf{p}_{\mathbf{r}}$ and $\mathbf{f}_{\mathbf{r}}$ have the same dimension. Knowing the coordinates of the virtual sticks in the end-effectors reference frames, $\mathbf{p}_{\mathbf{a}}$ and $\mathbf{p}_{\mathbf{r}}$ can be expressed in function of the position and orientation of the end-effectors $\left(\mathbf{O}_{\mathbf{h} 1}\right.$ and $\left.\mathbf{O}_{\mathbf{h} 2}\right)$, and $\mathbf{f}_{\mathbf{a}}$ and $\mathbf{f}_{\mathbf{r}}$ can be expressed in function of the forces and moments at the endeffectors level. The details of these calculations can be found in [3] but are not relevant for the comprehension of this paper.

\section{B. Assembly Task}

Dauchez proposed to extend the idea recalled above to the assembly of two objects [5]. When the two objects to be assembled are in contact, they can be considered as a single object. However they will move with respect to each other during the assembly phase and therefore a unique reference point $\mathbf{O}_{\mathbf{a}}$ cannot be defined anymore (otherwise the tips of the virtual sticks would not coincide anymore during the motion and the assumption of small discrepancy between these tips used in [3] would not hold). To cope with this problem, it is proposed in [5] to initialize the virtual sticks when the objects come in contact as the vectors joining $\mathbf{O}_{\mathrm{h} 1}$ to $\mathbf{O}_{\mathrm{a}}$ and $\mathbf{O}_{\mathrm{h} 2}$ to $\mathbf{O}_{\mathbf{a}}$, where $\mathbf{O}_{\mathbf{a}}$ is now the initial contact point. The contact is detected by using the wrist force sensors of the arms.

The idea is then that knowing the desired final relative configuration of the end-effectors, we can compute a trajectory for $\mathbf{p}_{\mathbf{r}}$ while keeping $\mathbf{p}_{\mathbf{a}}$ constant: the two arms will move symmetrically about $\mathbf{O}_{\mathbf{a}}$. To do so, it is necessary to redefine the virtual sticks at each sampling time by affecting half of the displacement to each stick (Fig. 2). For instance, if an elementary relative displacement $\Delta \mathrm{y}$ at time $\mathrm{t}$ is performed along the $\mathbf{y}$ axis of a reference frame centered in $\mathbf{O}_{\mathbf{a}}$, the new virtual sticks after this motion will be:

$$
\begin{aligned}
& \boldsymbol{l}_{\boldsymbol{h} 1}(t+\Delta t)=\boldsymbol{I}_{\boldsymbol{h} 1}(t)+\Delta y / 2 \boldsymbol{y}, \\
& \boldsymbol{l}_{\boldsymbol{h} 2}(t+\Delta t)=\boldsymbol{I}_{\boldsymbol{h} 2}(t)+\Delta y / 2 \boldsymbol{y} .
\end{aligned}
$$

More generally, equations of types (1) and (2) allow to determine the virtual sticks completely and at each step, and to perform various kinds of assemblies. The symmetry of these equations represent the fact that the reference frame centered in $\mathbf{O}_{\mathbf{a}}$ is invariant. In other words, this frame is the pivot about which the assembly is performed. However, before beginning the assembly, it is necessary to bring the two objects in contact with desired position and orientation. A method proposed by Dauchez in [6] is summarized in the next section.

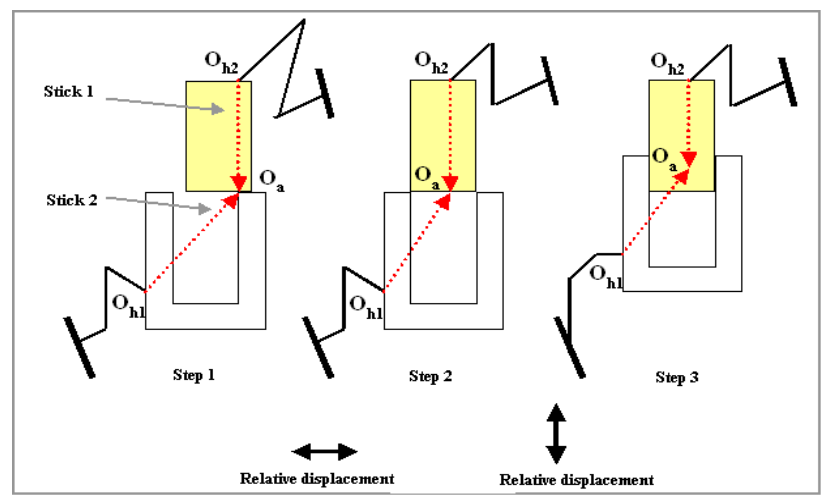

Fig. 2 Evolution of the virtual sticks during an assembly task "in space" 


\section{RELATIVE POSITIONING OF TWO MANIPULATORS}

\section{A. General Idea}

When a manipulator has more degrees of mobility than the number of constraints imposed by the task to be performed, we know this induces control problems but also that it is a great advantage in terms of success of the task (redundancy). When using a two-arm robot, it is therefore obvious that we should take advantage of the redundancy of the system. In the assembly task we are considering in this paper, once the objects are grasped, the first step is to bring them to a contact configuration, from which the assembly phase will start, using for instance the method summarized in section II (which again is our choice). If we impose the absolute position/orientation where this contact should occur, we are imposing additional and non-necessary constraints. We may even have too many constraints and find no solution to a problem which is theoretically solvable. In other words, the operator should not intervene and disturb the robotic system in such a task as to bring the end-effectors of two manipulators in a given relative position/orientation. Therefore, the solution proposed by Dauchez in [6] was to consider the two arms as a single manipulator, the base frame of which would coincide with the tip frame of one of the arms. This is detailed in the next paragraph.

\section{B. Calculations}

Let us attach two reference frames to the end-effectors of the arms, namely R0 and R1 (Fig. 3). The approach phase of an assembly task "in space” consists in bringing R0 and R1 in a given relative position and orientation. To do so, we propose to consider $\mathrm{R} 0$ as the base frame and $\mathrm{R} 1$ as the tip frame of a single manipulator composed of the two arms. Thus the problem is reduced to defining the trajectory of R1 with respect to R0 for a n-degree-of-freedom robot, $n$ being the sum of the degrees of freedom of each arm. If $n$ is greater than the number of constraints imposed by the task, we can use this redundancy to minimize some criterion (such as the range of motions of the joints) of satisfy some additional constraints (such as to leave a joint motionless).

On another hand, one important feature of this approach is that since the two arms will move during the task, we do not know in advance where the "rendez-vous" will occur. It actually depends upon the initial configurations of the arms and of course upon any criterion or constraint we would like to impose. This means that we do not have to define it in a common workspace, which can be complicated, and that the chances of success are higher, since in some sense the operator does not disturb the system in imposing the rendezvous location. In terms of trajectory execution, the easiest solution is to use the differential model:

$$
\Delta \boldsymbol{X}=\boldsymbol{J}(\theta) \Delta \theta
$$

where $\Delta \mathbf{X}$ is a vector comprising the variations of position and orientation of $\mathrm{R} 1$ with respect to $\mathrm{R} 0, \Delta \theta$ is the vector of the corresponding variations of the $\mathrm{n}$ joint variables and $\mathbf{J}$ is the Jacobian matrix of the single kinematic chain consisting of the two arms.

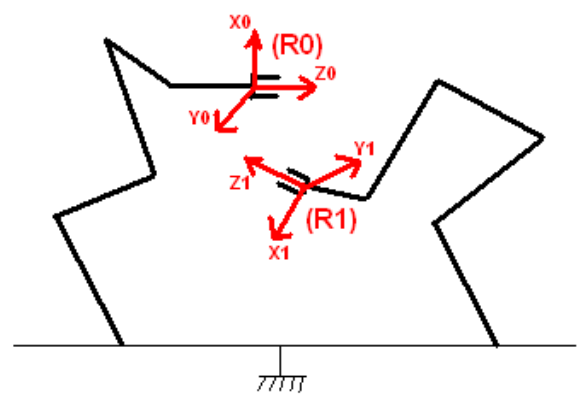

Fig. 3 Base and tip frames for a relative positioning of two manipulators

$\Delta \mathbf{X}$ represents the relative trajectory, determined at time $\mathrm{t}$ by the difference between a desired relative position/orientation $\mathbf{X}_{\mathbf{d}}$ and the actual position/orientation $\mathbf{X}_{\mathbf{a}}$ at same time $t$ (Fig. 4). To perform this motion, we calculate the corresponding joint motion $\Delta \theta$ by inverting (3) with the pseudo-inverse $\mathbf{J}^{+}$of $\mathbf{J}$ :

$$
\Delta \theta=J^{+} \Delta X
$$

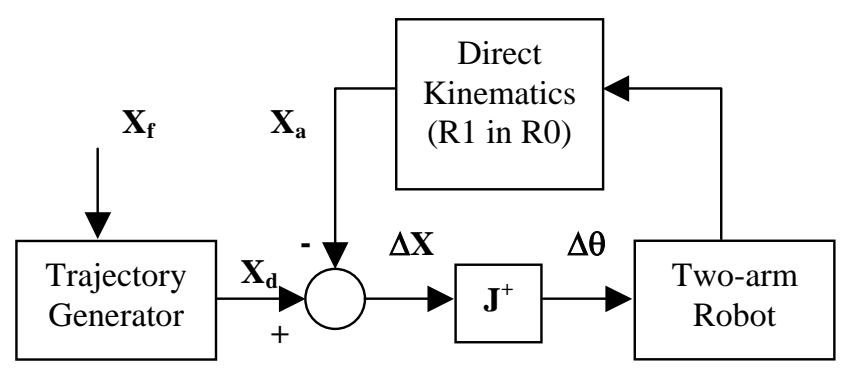

Fig. 4 Kinematic control for the relative positioning of two manipulators

It is of course possible to add some terms on the right hand side of (3) as long as they belong to the null space of $\mathbf{J}$ [6]. Fig. 4 summarizes the entire approach in the form of a block diagram. It shows that a trajectory generation algorithm must be used. It should generate a sequence of desired vectors $\mathbf{X}_{\mathbf{d}}$ with a step small enough to allow the use of the linearized model (3). $\mathbf{X}_{\mathbf{f}}$ is the desired final position/orientation of R1 with respect to $\mathrm{R} 0$.

\section{HEAD/ARMS POSITIONING OF A HUMANOID ROBOT (HRP2)}

\section{A. The simulator}

The HRP consortium has developed a software named OpenHRP (Open architecture Humanoid Robotics Platform) 
$[7,8]$. It allows the user to develop new controllers for the HRP2 humanoid and to test them using a virtual environment. The software is entirely compatible with the real robot: if the simulation works, the real experiment will be a success too (at least if all the mechanical and control aspects of the real humanoid are as perfect as the simulated ones). OpenHRP uses the VRML language (Virtual Reality Modeling Language) to model the robot and its environment. Generally speaking, this language allows the user to create $3 \mathrm{D}$ virtual worlds and share them on the Internet. A special norm, Hanim [9] has been developed for humanoids. The kinematic and dynamic relationships of the different parts of such a robot (neck, shoulder, arm, etc.) can be defined. This is what we used to simulate our algorithms with the HRP2 robot, keeping in mind that we are interested in the arms and the head (for the vision system) of this humanoid for assembly tasks. Therefore we considered two cameras on the head, two wrist force sensors and two grippers. Each arm has three joints at the shoulder level, two at the elbow level and one at the wrist level. Fig. 5 shows sketches of our VRML model of HRP2.
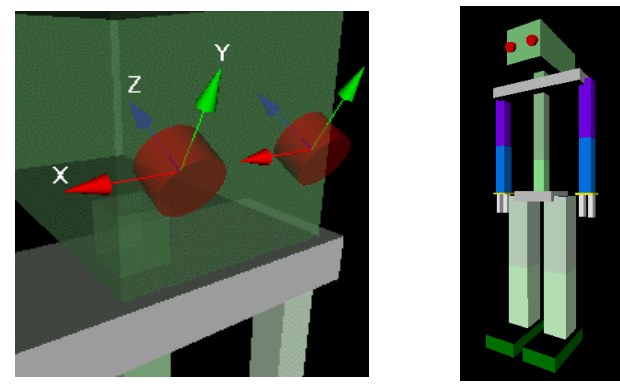

Fig. 5 VRML model of a humanoid with the same properties as HRP2

With this simulation tool, we have successfully performed experiments on the relative positioning of the arms, using the method described in section III and taking into account the various joint limits. Then we have tested a solution to keep the end-effectors visible from the eyes, i.e. to servo the head's position such as to look at the hands. This is explained in the next paragraphs.

\section{B. Positioning the head}

Section II has summarized a solution to perform an assembly "in space" with a two-arm system. Section III has proposed an automatic method to bring the end-effectors to a given relative configuration. However to combine these two approaches and therefore realize an assembly automatically, it is necessary to determine the contact point $\mathbf{O}_{\mathbf{a}}$ between the two objects at the end of the approach phase. Since we do not necessarily know the exact dimensions of the objects or their positions within the grippers, the vision system of the humanoid might be helpful. Because the approach summarized in Section III does not specify the "rendez-vous" location, it is necessary that the vision system of the robot (i.e. the head) follow the arms during their motions. More precisely, we propose here that the head be always aligned with a reference frame centered at a point $\mathrm{M}$ located in the middle of the end-effectors (Fig. 6). This should allow the robot to see the objects at the end of the approach phase.

At a given time, from the joint measurements of both arms, we can compute the Cartesian position and orientation of the end-effectors through the direct kinematic models. From these data, we can determine what the pitch, roll and yaw angles of the head should be in order to see an area located in the middle of the end-effectors, as explained below.
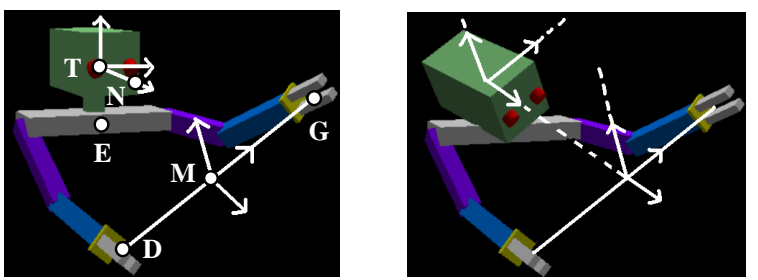

Fig. 6 Position servoing of the head

\section{Computing the Head's Roll (r), Pitch (p) and Yaw (y)}

To perform these calculations, we can consider three planar problems. In the three figures below, points T, N, E, D, M, G are those defined in Fig. 6. O is only an intermediate point used for calculation purpose but does not represent the same actual point in the three cases.

\section{ROLL (Fig. 7)}
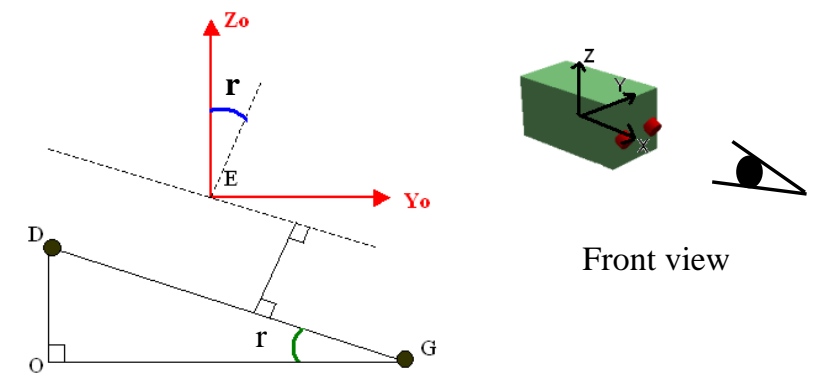

Front view

Fig. 7 Geometric relations for the calculation of $r$

Cartesian coordinates of O, D and G are known or can be computed from the joint values of each arm. From Fig. 7, we have:

$$
\cos (r)=\mathrm{OG} / \mathrm{DG}
$$




\section{PITCH (Fig. 8)}

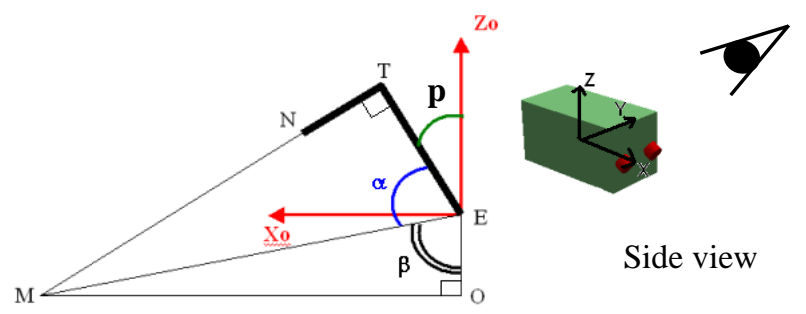

Fig. 8 Geometric relations for the calculation of $p$

TE is constant and known (user-defined points on the robot). Cartesian coordinates of $\mathrm{E}, \mathrm{M}$ and $\mathrm{O}$ can be computed from the robot's configuration. From Fig. 8, we have:

$$
\begin{gathered}
\cos (\alpha)=\mathrm{TE} / \mathrm{ME} \\
\cos (\beta)=\mathrm{OE} / \mathrm{ME} \\
p=\pi-\alpha-\beta
\end{gathered}
$$

\section{YAW (Fig. 9)}

Cartesian coordinates of T, E, M and O are known or can be computed from the robot's configuration. From Fig. 9, we have:

$$
\cos (y)=\mathrm{OE} / \mathrm{ME}
$$
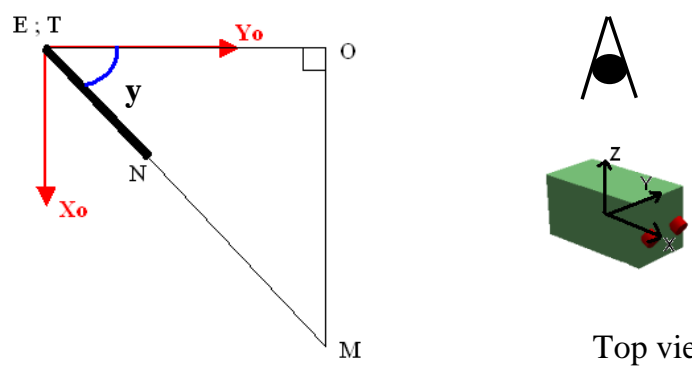

Top view

Fig. 9 Geometric relations for the calculation of $y$

Equations 5, 8 and 9 give us the roll, pitch and yaw angles the head should have in order to follow point $\mathrm{M}$, whose coordinates are calculated from the direct kinematic models of each arm. We have tested this algorithm with OpenHRP and have successfully servoed the motion of the head on the motion of the arms during an approach phase based upon the solution presented in section III.

\section{Remarks}

Since the ideas developed in sections III and IV only involved some mathematics, we have actually first implemented them on Matlab ${ }^{\circledR}$ an have thus determined for several experiments a file named "angle.dat" which includes the 12 joint variables of the two arms and the 3 joint variables of the head at each sampling time. The sampling period was $0.002 \mathrm{~s}$ and the total simulation time $10 \mathrm{~s}$ (5000 points). Then the controller implemented on the simulator OpenHRP reads the joint values in "angle.dat" and uses them for display (Fig. 10).

Once a simulation is completed, 3D video segments of the scene can be created and the time evolution of any joint variable of the humanoid can be observed and analyzed.

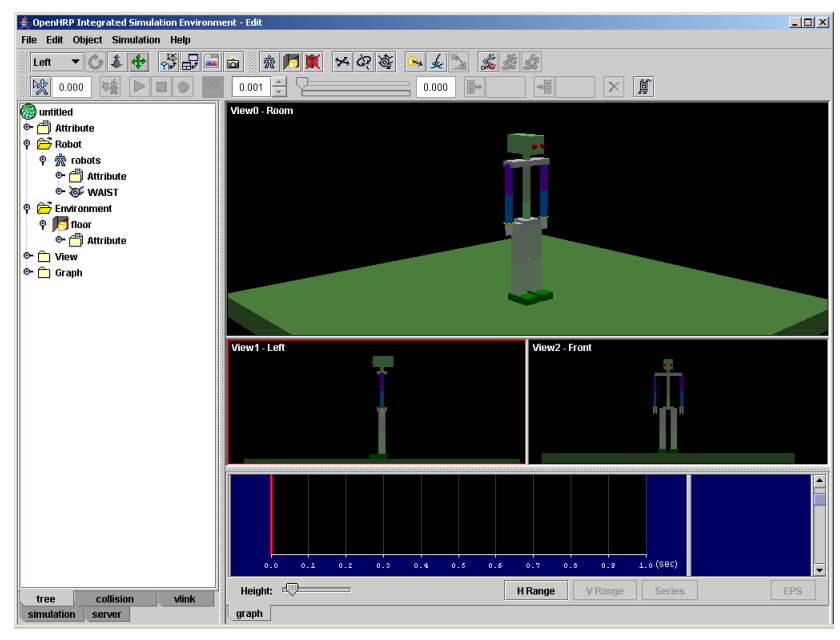

Fig. 10 Simulation under OpenHRP

\section{CONCLUSIONS}

This introductory paper was humbly aimed at presenting various steps of an assembly task performed by a humanoid robot, namely HRP2:

- relative positioning of the head and the arms with the help of a vision system,

- relative motion of the objects until a contact occurs,

- assembly itself through a symmetric position/force control scheme.

For each of these steps, a method has been summarized but in this preliminary work, only the relative positioning of the arms and that of the head and the arms have been tested with the HRP2 simulator. This is partly due to the fact that we had access to a version of the simulator that is available at no cost on the Internet and that did not allow us to include vision or force data. Therefore, the grasping of the objects has not been mentioned here either, since it would require image processing algorithms. Some solutions to this problem can be found in the literature $[9,10]$. 
In addition, we have to admit that we have addressed a "limited" problem and have not considered the general question of keeping the hands' workspace visible throughout the task. This raises for instance the issue of limits or singularities of the head's joints. This should also be explored in more detail in the future.

The natural next steps of this work is hence to further investigate some theoretical issues, to combine all the algorithms summarized in this paper, and to perform a complete assembly task, first in simulation, and then hopefully on the real robot, if available. This should be feasible because of the existence of the Japanese/French JRL (Joint Robotics Laboratory) which is focusing its activities on the HRP2 humanoid robot.

Obviously, in the context of manipulation tasks, one has to admit that there could be some parameters to adjust to go from simulation to real experiments. The authors have a solid experience of demonstrations with real manipulators and are perfectly aware of the gap between computer simulation and prototyping. Therefore they strongly believe they could succeed in performing automatic complex assembly tasks with a humanoid robot in the years to come.

\section{REFERENCES}

[1] K. Kaneko et al. "Design of Prototype Humanoid Robotics Platform for HRP," Conference on Intelligent Robots and Systems, Lausanne, Switzerland, October 2002, pp. 2431-2436.

[2] M. Raibert and J. Craig, "Hybrid Position/Force Control of Manipulator," Trans. ASME, Journal of Dynamic Systems, Measurement, and Control, vol. 103, no. 2, 1981, pp. 126-133.

[3] M. Uchiyama and P. Dauchez, "A symmetric Hybrid Position/Force Scheme for the Coordination of Two Robots," IEEE Conference on Robotics and Automation, Philadelphia, Pennsylvania, USA, April 1988, pp. 350-356.

[4] S. Hayati, K. Tso, and T. Lee, "Generalized Master/Slave Coordination and Control for a Dual Arm Robotic System," Second International Symposium on Robotics and Automation, Albuquerque, New Mexico, USA, November 1988, pp. 421-430.

[5] P. Dauchez and X. Delebarre, "Force-Controlled Assembly of Two Objects with a Two-Arm Robot,” ROBOTICA, vol. 9, 1991, pp. 299-306.

[6] P. Dauchez, R. Zapata and P. Coiffet, "Cooperation of Robots in Gripping Tasks: the Exchange Problem,” ROBOTICA, vol. 1, 1983, pp. 73-77.

[7] F. Kanehiro et al., "Open Architecture Humanoid Robotics Platform," IEEE Conference on Robotics and Automation, Washington, D.C., USA, May 2002, pp. 24-30.

[8] http://www.ist.aist.go.jp/humanoid/openhrp/

[9] http://h-anim.org

[10] S. Kagami, JJ. Kuffner, N. Nishiwari, "Humanoid Arm Motion Planning Using Stereo Vision and RRT Search,” Conference on Intelligent Robots and Systems, Las Vegas, Nevada, USA, October 2003, pp. 2167-2172.

[11] C. Borst, M. Fischer, S. Haidacher, H. Liu, G. Hirzinger, "DLR Hand II: Experiments and Experiences with an Anthropomorphic Hand," IEEE Conference on Robotics and Automation, Taipei, Taiwan, September 2003, pp. 702-707. 